\title{
Back analysis of reinforced soil slopes
}

\author{
P. Procházka ${ }^{1} \&$ J. Trckova ${ }^{2}$ \\ ${ }^{1}$ Czech Technical University in Prague, Czech Republic \\ ${ }^{2}$ IRSM, Czech Academy of Sciences, Prague, Czech Republic
}

\begin{abstract}
Among the most popular reinforcement in soil mechanics of slopes is anchoring and nailing. In our experiments nails are applied; they are penetrated into the slope, it is loaded only by its volume weight. The material of the nails, as well as that of the slopes, is known from laboratory tests. This circumstance influences the distribution of stresses along the length of the body of the nail. Moreover, the position of the nails to the stability of the reinforced slopes is observed. As the experiments are carried out in scale models, similarity conditions have to be obeyed. The technology of construction of experimental models is very important. Similarity rules are applied, but in this case no additional tests on physical equivalence of materials (real and that in the scale model) are necessary. As is well known that slope stability is a phenomenon which underlies the softening material behavior, i.e. the nonlinear behavior is concentrated along the slip curve. All kinds of nails are fully active after their mobilization. A different position of nails is considered to obtain the influence of this effect.

In numerical analysis and a priori integration method is fully used. Its application enables one to decide relatively quickly if the slope is stable or the measure of stability, the safety margin. Originally, the method was applied to stability of both homogeneous and nonhomogeneous slopes, streaming water and pore pressure influence on the slope stability. Here the influence of nails is considered by additional slip force along the slip surface. The force is calculated from comparison with the experimental data. The nails are introduced in such a way that they are long enough to cross the most dangerous slip curve possible.

Keywords: nailed slopes, scale models, a priori integration method, back analysis.
\end{abstract}




\section{Introduction}

The concept of a priori integration of functionals of the classical method of slope stability assessment was published for the first time in [1] for the classical plain gravity model. Before these dates it was applied to the analysis of slope stability for the Prague Underground Railway and formed the subject of several thematic problems and improvement suggestions. Further possibilities of the method have been shown also in the solution of the earth pressure problem and a wide range of applications is found in [2].

The formulation of the method is based on the combination of variation problems with the strip methods. In practice these slice methods depend on a single argument only and make it possible to express the functionals in the variation formulation in the form of functions. If we consider the very accurate interpretation of the values of functions by contemporary computers, it is obvious that a priori integration results not only in the acceleration of computer processing of stability problems, but also in increased accuracy of computation and subsequent determination of the form of the permissible shear surface and the safety factor value with any accuracy required. The error of the method resulting from introduced assumptions will not be eliminated, naturally, but will be reduced by the possible introduction of better contact assumptions.

Coupled problem have been solved in a couple of papers by Procházka and Trckova [3, 4]. Here internal parameters for a back analysis served design parameters of some optimization problems. The modeling from physically equivalent materials in stands (scale models) starts with papers [5-7]. Application of back analysis to stability of tunnel structures is described in [8], in [9], the stability of tailings (deposits) from open-pit mines is solved and in [10]. Identification of internal parameters using back analysis is studied in [11].

\section{Basic principles of a priori integration method (AIM)}

The idea of the AIM arose from the needs of design practice. In the design of big excavations or embankments which occurred, e.g., in the construction of the underground railway it was found suitable to base the actual design on parametric studies depending on the simplified geometry of the slope and the geotechnical parameters of the soil of which the slope consists. Computations have shows explicitly that for reasons of final assessments of slope stability it was impossible to use modern numerical methods (finite element method, boundary element method). However, classical slice methods did not appear entirely suitable, either. Modern methods need more computational time, while slice methods are numerically unstable and do not enable the application of minimization strategy for a more accurate stability coefficient computation.

One of the possibilities of elimination of these shortcomings consists in the application of the AIM, e.g., to the classical plain gravity model which has the advantage of explicit expression of the stability coefficient on the given shear surface, can easily be extended to three dimensions and involve other influences on the slopes. 
Before presenting the initial formulas we introduce some symbols and assumptions. First we will deal with two-dimensional problems in $O x y$ coordinate system. In practice it is advantageous to locate the origin of the coordinates $O$ at the toe of the slope. The application of the AIM consists in expressing the problem in functional form. For the classical model, for instance, we seek the stability measure (safety factor) on a concrete admissible shear surface with the understanding that the safety factor is the minimum of stability measures. Moreover, in the AIM we express the functionals for a fixed shear surface in the form of functions. This can be achieved, e.g., with the assumption (frequently used in engineering practice) that geotechnical parameters are homogeneous and isotropic by parts (by layers). In our case homogeneous medium is considered, as the influence of nails is studied in the scale model.

Let $y=t(x)$ be the boundary of the slope surface (terrain) and $y=f(x)$ describe the shear surface the admissible form of which is a part of the circle. In order not to complicate the explanation, let us assume that $f$ is a function, i.e. that there is just one value of $y$ for every $x$ within the admissible interval. The generalization of this assumption is not connected with any difficulties. Further, in accordance with the principal idea of the model (equilibrium on the fixed shear surface together with the respective denotations is shown in Fig. 1) it is possible to define the safety factor $F$ on the shear surface as follows:

$$
F=\frac{N \tan \varphi+C}{T}
$$

where $N$ and $T$ are the normal and tangential components, respectively (with reference to the shear surface) of the unit weight of the soil above the shear surface, $\varphi$ is the angle of internal friction (shearing resistance) and $C$ is the cohesion.

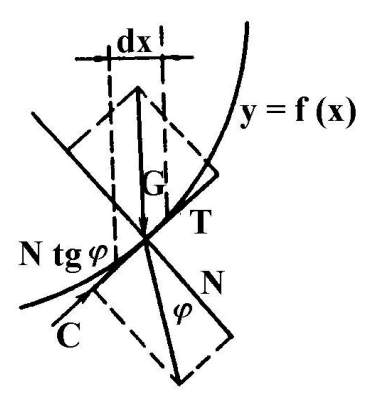

Figure 1: Equilibrium of forces on the shear surface in the gravity model.

The most probable location of the shear surface and simultaneously the safety factor value (minimum safety factor) are determined by the minimization of the values of $F$, i.e. 


$$
F_{0}=\operatorname{minimum} F
$$

where the minimum is considered across all admissible shear surfaces, i.e. such circles for which the set of those $x$ for which

$$
S(x)=P_{+}[t(x)-f(x)] \neq 0
$$

is not empty. In the definition of the function $S$ we have introduced the Heaviside operator

$$
P_{+}[a]=1 \text { for } a>0, \quad P_{+}[a]=0 \quad \text { otherwise }
$$

The condition (3) means that the diagrams of the functions $t$ and $f$ and intersect at least in two points and $t\left(x_{0}\right)>f\left(x_{0}\right)$ at least for one $x_{0}$. The condition (2) determines the form (or, to be exact, the location) of the shear surface along which the slip will occur most probably, if the safety factor $F_{0}$ is lower than the respective safety factor of the slope, determined either by a standard (EC7-1, DIN, CSN), or by the designer's experience. If the safety factor is higher than this number, the slope can be considered stable.

Now we can express the individual terms in (1) as follows:

$$
\begin{gathered}
T(f)=\int_{-\infty}^{\infty} \int_{f(x)}^{t(x)} \gamma(x, y) p(x) S(x) \mathrm{d} y \mathrm{~d} x, \quad C(f)=\int_{-\infty}^{\infty} \frac{c(x)}{q(x)} S(x) \mathrm{d} x \\
N \tan \varphi(f)=\int_{-\infty}^{\infty} \int_{f(x)}^{t(x)} \gamma(x, y) \tan \varphi(x) q(x) S(x) \mathrm{d} y \mathrm{~d} x
\end{gathered}
$$

where $\gamma$ is the volume weight of the soil, $c$ is the cohesion, and

$$
p(x)=\frac{x-x_{C}}{R}, q(x)=\sqrt{1-p^{2}}
$$

where $\left(x_{C}, y_{C}\right)$ are the coordinates of the center of the slip surface and $R$ is its radius. Note that the functions $p$ and $q$ in (6) are the sine and cosine, respectively of the angle $\alpha$ between the tangent to the slip surface at the point $\left(x, \sqrt{R-\left(R^{2}-\left(x-x_{C}\right)^{2}\right.}\right)$ and the axis $x$ (see Fig. 2). The material constants $\varphi$ and $c$ can be entered as residual or peak values. In this way it is possible to consider also the influence of deformation. Description of the geometry and subregions, the procedure of the AIM can be applied, is seen from Fig. 2. Roman numerals denote homogeneous and isotropic subdomains (elements), Arabic 
numerals describe the vertices of element boundaries. It can easily be verified that the formulas (5) correspond with the relations of the gravity model for the case of limit transition in the meaning of the Riemann integral definition.

The cases most frequently occurring in practical computations are the cases of soil mass, the boundary of which can be approximated by a polygon and the material of the mass is homogeneous and isotropic in parts, while these parts (subdomains) are also bounded by polygons.

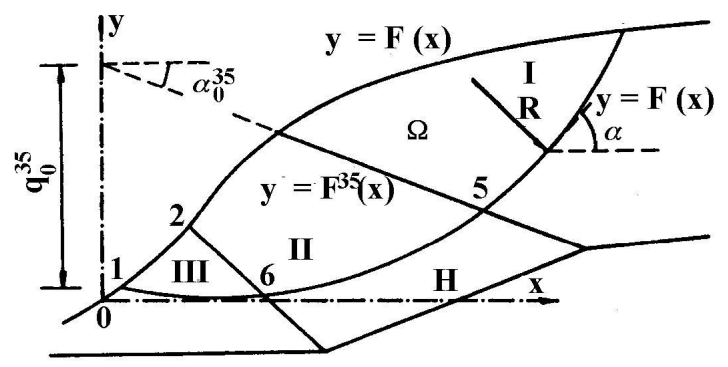

Figure 2: Topological relations of the AIM.

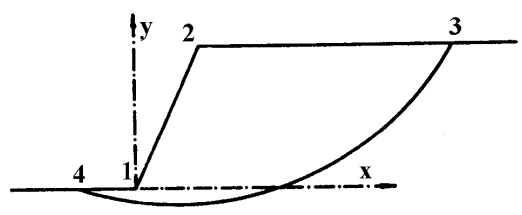

Figure 3: Geometry of the simple slope.

In order to study the influence of reinforcing nails, simple slope, i.e. a homogeneous isotropic slope without benches, see Fig. 3, is considered, and the slip curve meets the toe of the slope, i.e. points 1 and 4 are identical. This is the case of slopes with steeper toe angles. Our aim is the computation of the values of integrals (5) for the latter case. As in this particular case $\gamma, \varphi$ and $c$ are constant, so that it holds that

$$
\begin{gathered}
T=\gamma\left\{\int_{\mathrm{x}_{1}}^{\mathrm{x}_{2}} t^{12}(x) p(x) \mathrm{d} x+\int_{\mathrm{x}_{2}}^{\mathrm{x}_{3}} t^{23}(x) p(x) \mathrm{d} x-\int_{\mathrm{x}_{3}}^{\mathrm{x}_{1}} f(x) p(x) \mathrm{d} x\right\} \quad C=c \int_{x_{1}}^{x_{3}} \frac{\mathrm{d} x}{q(x)} \\
N \tan \varphi=\gamma \tan \varphi\left\{\int_{\mathrm{x}_{1}}^{\mathrm{x}_{2}} t^{12}(x) q(x) \mathrm{d} x+\int_{\mathrm{x}_{2}}^{\mathrm{x}_{3}} t^{23}(x) q(x) \mathrm{d} x-\int_{\mathrm{x}_{3}}^{\mathrm{x}_{1}} f(x) q(x) \mathrm{d} x\right\}
\end{gathered}
$$

where superscripts $i j$ stands for abscissa or circle of the slip line $i-j, i, j=1,2,3$. Furthermore, in the last equations $x_{i}$ and $y_{i}$ are coordinates of point $i$. Substituting for the slope $1-2$ and ridge $2-3$ yields 


$$
t^{12}(x)=k_{0}, \quad t^{23}(x)=q_{0}, \quad f(x)=y_{C}-R q(x)
$$

where $k_{0}$ is the value of the slope and $q_{0}$ is the height of the ridge. We ascertain that the explicit expressions of $T, N \tan \varphi$ and $C$ is split into an algebraic sum of influences of individual abscissas forming the boundary of the slope and its individual layers and the parts of the circles forming the slip surface.

Before coming to the explicit expression of influences from the integration over an abscissa or a circle it is advisable to introduce substitution (parameter $p$ stands for sine of the angle $\alpha$ ) and formulate functions:

$$
\begin{aligned}
& x=x_{C}+p R \quad \mathrm{~d} x=R \mathrm{~d} p \quad F_{1}(p)=\frac{\left(\sqrt{1-p^{2}}\right)^{3}}{3} \quad F_{2}(p)=\frac{p^{2}}{2} \\
& F_{3}(p)=\frac{\arcsin p+p \sqrt{1-p^{2}}}{2} \quad F_{4}(p)=\frac{p^{3}}{3} \quad F_{5}(p)=p-\frac{p^{3}}{3} \quad F_{6}(p)=\arcsin p
\end{aligned}
$$

Now it easily follows that the influences from integrations below the abscissas can be expressed as (denoted by brackets):

$$
[T]=\left[A F_{4}(p)+B F_{2}(p)\right]_{i}^{j} \quad[N \tan \varphi]=\left[B F_{3}(p)-A F_{1}(p)\right]_{i}^{j} \tan \varphi
$$

where

$$
A=\gamma R^{2}, B=\gamma R\left(k_{0} x_{C}+q_{0}\right), \text { and }[f(p)]_{i}^{j}=f\left(p_{j}\right)-f\left(p_{i}\right), p_{i}=\left(x_{i}-x_{C}\right) / R
$$

In case of circular segments are considered the following formulas can be derived:

$$
[T]=\left[A F_{2}(p)+B F_{1}(p)\right]_{i}^{j} \quad[N \tan \varphi]=\left[A F_{3}(p)-B F_{5}(p)\right]_{i}^{j} \tan \varphi \quad C=c R\left[F_{6}(p)\right]_{i}^{j}
$$

where $A=\gamma R y_{C}, B=\gamma R^{2}$. The loading from above the ridge is taken into volume weight, while the influence of the nail (nails) is an additional shear force at the cross of current slip circle and the nail. The value of it follows from the experiments. If instead of a nail a geotextilie is put into the material of the slope, one force is needed to explain the influence of the reinforcement on the stability of the slope. This is not the case, so that a different number of nails is necessary to consider. The aim of the coupled modeling consists in tuning the numerical model, turned to a programming code, in such a way that the influence of the nails can be identified with high accuracy. 


\section{Physical modeling}

Physical modeling is important for the study of effects taking place in soil mass in connection with construction of underground structures. The modeling allows us to investigate mechanisms of geotechnical phenomena, predicts stress changes and their demonstration during various progress of underground construction and also during simulation of operating conditions.

Basic rules of the experimental modeling and formulation of the mechanical conditions for modeling come from the principles of geometrical and physical similarity which is inferred for a consideration of dimensional analysis, [6-8]. According to the Buckingham theorem, [6], the dimensional equation for the relation between reality and the model can be reduced to the problem of finding relevant non-dimensional parameters. Equations can be determined, their arguments are dimensionless.

The physical model has to obey geometrical similarity; this is the proportionality of dimensions and the identity of angles between the model and modeled object in the whole range of the model.

The type of modeled geotechnical problem and its extent, possibility and technique of bringing forces, time factor and other aspects, technical possibilities, assign linear scale of the model. It is the aspect ratio in which length dimensions of the model are reduced against the reality. Note that the similarity being valid for slope stability assessment can be found in [11]. The models are constructed from mixture of various, mostly easy available materials (e.g. sand, bentonite, ballotine, gypsum, mica - vermiculite, composite mortar, cellular concrete and water).

The models are constructed in stands of various dimensions in dependence of solving problem and scale of the model. Compact structure of the stand is formed by reinforced frame performing no deformation of it during the model test. Front wall of the model stand can be glassed to observe and measure deformation of the model.

\section{Example}

Slope 1:1.5 (length:height) is considered in the scale of 1:100. The slope obeys the similarity laws according to the previous section. Physically equivalent material is selected to be in compliance with selected real slope as: Mixture of ballotine and fat is the basic material for the laboratory tests in stands, the contents of ballotine was $99.87 \%$, fat A00 was $0.125 \%$. As the filling quartz sand with granularity of 1-7 mm was used. The stand is $1.5 \mathrm{~m} \mathrm{x} 1 \mathrm{~m}$ x $0.5 \mathrm{~m}$. Material properties of the slope are listed below:

$\begin{array}{ll}\text { Volume weight } & 1.707 \mathrm{~g} / \mathrm{cm}^{3} \\ \text { Cohesion } & 0.722 \mathrm{kPa} \\ \text { Angle of internal friction } & 24^{\circ} \\ \text { Oedometric modulus } E & 5560 \mathrm{kPa} \\ \text { Coefficient of compressibility } & 152 \\ \text { Tensile strength } & 5.1 \mathrm{kPa}\end{array}$


Density of the sand

$1.93 \mathrm{~g} / \mathrm{cm}^{3}$

Density of saturated sand

$2.16 \mathrm{~g} / \mathrm{cm}^{3}$

In the following pictures stands with different stages of loading and reinforcements are shown. The loading from above the ridge was applied to tune the material properties of the aggregate in the model (the slope created from the physically equivalent material together with a nail). The nails have been prepared from a chip of bamboo, unified in the shape and dimensions, their shear strength is known, so that the resistance force due to a nail is also given. Influence of load to the safety margin is depicted in Fig. 4.

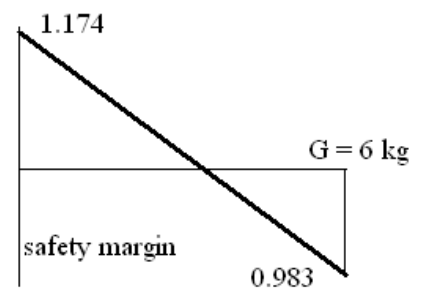

Figure 4: Relation load and safety margin.

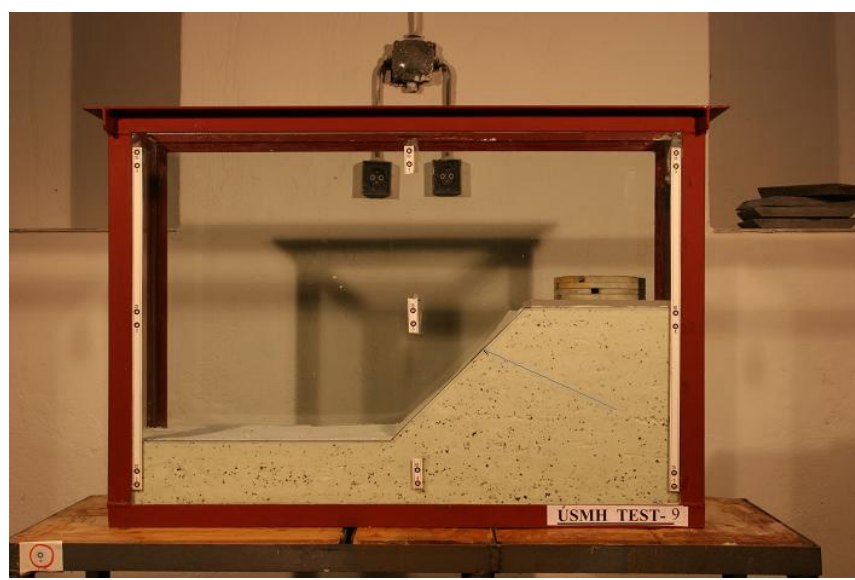

Figure 5: View of stand with the slope loaded from above and with the first position of a nail.

Two basic positions is horizontal set of nails were prepared. The first is seen in Fig. 5, it should help to stiffen the upper part of possible slip surface (curve). As is well known, this is the most advantages position, since the slopes in general start their damage along the ridge. The first loading step is also seen from this picture.

In Fig. 6 the second position of reinforcement is depicted together with obvious movement of the ridge. Also in the upper right part of the slope partial damage is highlighted and according to the assumed shape of the slip curve the safety margin is derived. 
It appears that for upper position of the nail one nail bears $12.29 \mathrm{~kg}$ load, two nails $16.15 \mathrm{~kg}$ and the necessary number of nails for higher value of the load can be extrapolated. For the lower position (second case) of the nail, one nail bears only $7.12 \mathrm{~kg}$, two nails $9.78 \mathrm{~kg}$ etc. The bearing capacity is considered for safety margin 1.3 , as most of standards consider.

It is worth noting that this approach for the identification of appropriate structure of slopes with reinforcement by nails can be applied to various types of slopes, even in non-homogeneous mediums. For this case and the AIM see [11].

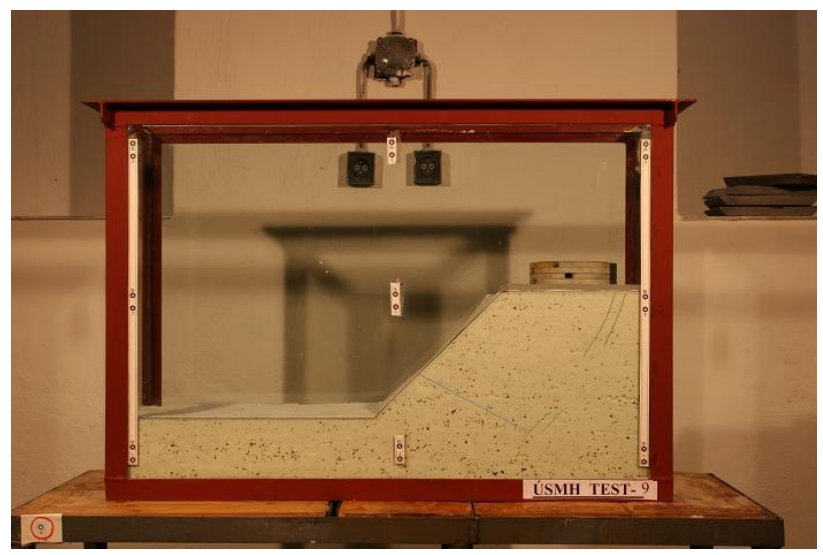

Figure 6: The second type of reinforcement, slip curves highlighted.

\section{Conclusions}

Coupled modeling, experimental in stands and numerical using the AIM is shown on model examples of reinforced slopes by nails. This simple algorithm involves very user-friendly algorithm of the AIM and relatively simple and cheap modeling in stands. Tuning of numerical models using results from experiments enables engineers to assess this type of structures with relatively high accuracy and very fast.

\section{Acknowledgement}

This paper was prepared under financial support of GA AV ČR, project No. IAA 2119402.

\section{References}

[1] Procházka, P., Koudelka, P.: Slope stability analysis by the Apriori Integration Method (in Czech), Inzenyrske stavby, Vol. 28/3, 79-84

[2] Koudelka, P., Prochazka, P.: Apriori Integration Method, Analysis, Similarity and Optimization of Slopes, Academia 2001, Prague 
[3] Procházka, P., Trcková, J.: Assessment and Control of Tunnel Structures based on coupled modelling. Submitted to Geotechnique

[4] Procházka, P., Trčková, J.: Coupled modeling of structural strength. Boundary Elements XXVII, eds. A. Kassab, C.A. Brebbia, E. Divo, and D. Poljak. Orlando, USA, WIT Press 2005, 155-164

[5] Buckingham, E.: On physically similar systems: Illustrations of the use of dimensional equations. The Physical Review, Vol. IV, series II. No. 4, 1914, 345-376

[6] Head, K.H.: Manual of Soil Laboratory Testing. John Wiley \& sons. Inc. New York, Toronto, 1992

[7] Kožešník, J.: Theory of similarity and modeling, Academia, Prague, 1983

[8] Procházka, P., Trčková, J.: Coupled modeling of Concrete Tunnel Lining, Our World in Concrete and Structures, Singapore, 2000, 125-132

[9] Procházka, P., Trčková, J.: Material properties of tailings of open-pit mines using coupled modeling, Proc. 5th European Conf. on Numerical Methods in Geotechnical Engineering NUMGE, ed. Mestat, Presses de IÉNPC/LCPC, Paris, 273-278, 2002

[10] Procházka, P., Trčková, J.: Identification of material parameters in underground structures, Proc. Material Characterization, ed. C.A. Brebbia, Santa Fee, NM, USA, 2003

[11] Procházka, P.: Slope optimization by the Apriori Integration Method, Acta Montana, IG CSAS, 82, 51-154, 1990 